\title{
HPIP promotes gastric cancer cell proliferation through activation of cap-dependent translation
}

\author{
BING CHEN $^{1,2}$, JIN ZHAO ${ }^{2}$, SHENGBIN ZHANG $^{2}$, YONGGANG ZHANG ${ }^{3}$ and ZONGHAI HUANG ${ }^{1}$ \\ ${ }^{1}$ Department of General Surgery, Zhujiang Hospital, Southern Medical University, Guangzhou, Guangdong 510282; \\ ${ }^{2}$ Department of General Surgery, The Third Affiliated Hospital, Inner Mongolia Medical University, \\ Baotou, Inner Mongolia 014000; ${ }^{3}$ Department of General Surgery, Affiliated Renmin Hospital of \\ Inner Mongolia Medical University, Hohhot, Inner Mongolia 010059, P.R. China
}

Received April 14,2016; Accepted June 7, 2016

DOI: $10.3892 /$ or.2016.5157

\begin{abstract}
Cap-dependent translation has an essential role in the control of cell proliferation by initiating the translation of oncogenes involved in the regulation of cell cycle progression, such as cyclin D1, and its deregulation contributes to the development and progression of various types of cancers. Hematopoietic pre-B-cell leukemia transcription factor interacting protein (HPIP) was found to be overexpressed in gastric cancer (GC) tissues compared to normal tissues and to promote $\mathrm{GC}$ growth in vitro and in vivo. However, the mechanism by which HPIP promotes GC cell proliferation remains unknown. In the present study, we found that HPIP activated cap-dependent translation in an AKT/mTORC1 pathway-dependent manner. Blocking cap-dependent translation with 4EGI-1, a specific eIF4E/eIF4G interaction inhibitor, profoundly abrogated the ability of HPIP to promote G1/S phase transition and GC cell proliferation, while activation of cap-dependent translation by silencing 4E-BP1 expression significantly reversed the inhibitory effect of HPIP knockdown on GC cell proliferation. Furthermore, targeting translation initiation with 4EGI-1 effectively suppressed the ability of HPIP to promote gastric tumor growth in a xenograft mouse model in vivo. All these data indicate that HPIP promotes GC cell proliferation through positive regulation of cap-dependent translation and mproves our understanding of the underlying mechanisms involved in the regulation of GC cell proliferation by HPIP.
\end{abstract}

Correspondence to: Dr Zonghai Huang, Department of Surgery, Zhujiang Hospital, Southern Medical University, 253 Middle Gongye Avenue, Haizhu, Guangzhou, Guangdong 510282, P.R. China E-mail: doctorpd1@aliyun.com

Abbreviations: HPIP, hematopoietic pre-B-cell leukemia transcription factor interacting protein; GC, gastric cancer; eIF4E, eukaryotic translation initiation factor $4 \mathrm{E}$; 4E-BP1, eIF4E-binding protein 1 ; mTORC1, mammalian target of rapamycin complex 1

Key words: HPIP, gastric cancer, cap-dependent translation, 4E-BP1, AKT, mTORC1, cell proliferation

\section{Introduction}

Gastric cancer (GC), the fourth common malignant tumor type, ranks as the second leading cause of cancer-related mortality worldwide (1). Currently, the main therapies for GC treatment include surgery, radiation therapy and chemotherapy. However even following maximal trimodality therapies, the prognosis of advanced GC patients remains poor with a median overall survival (OS) of less than 12 months and a 5-year survival rate of less than $5 \%$ (2). Elucidating the molecular mechanisms underlying GC progression is essential for developing novel diagnostic and therapeutic strategies against this disease.

Cap-dependent translation plays a crucial role in the initiation and progression of many types of cancers by enhancing the translation initiation of many oncogenic mRNAs such as cyclin D1, Bcl-2, MMP9 and VEGF that regulate cell proliferation, survival, metastasis and angiogenesis, respectively (3). Cap-dependent translation is controlled by the eukaryotic translation initiation factor $4 \mathrm{~F}$ (eIF4F) complex, which comprises eIF4E, eIF4G and eIF4A and is essential for translation initiation by recruiting the 40S ribosome subunit to the 5' cap mRNA (4). The availability of eIF4E is the rate-limiting factor for the assembly and activity of the eIF4F complex and is controlled by the eIF4E expression level and the phosphorylation status of eIF4E-binding protein 1 (4E-BP1) (5). Unphosphorylated 4E-BP1 negatively regulates the formation of the eIF4F complex by competing with eIF4G for binding to eIF4E. Phosphorylation of 4E-BP1 releases its binding to eIF4E, allowing the binding between eIF4G and eIF4E and translation initiation (6). The activity of 4E-BP1 is mainly regulated by the mammalian target of rapamycin complex 1 (mTORC1), an important downstream target of AKT signaling. mTORC1 phosphorylates 4E-BP1 on Thr37 and Thr46, which facilitates subsequent phosphorylation of Ser65 and Thr70, leading to the dissociation of 4E-BP1 and eIF4E and the assembly of the eIF4F complex (7). eIF4E was reported to be overexpressed in gastric tumor tissues compared with that in adjacent normal tissues and silencing of eIF4E was found to inhibit the proliferation of GC cells (8). The AKT/mTORC1 pathway is frequently activated in GC and is directly associated with its progression (9). Several groups have reported higher expression of p-4E-BP1 in GC tissue 
samples than that noted in normal tissue samples (10-12). All of these studies indicate that cap-dependent translation contributes to gastric tumorigenesis and may serve as a potential therapeutic target for GC.

Hematopoietic pre-B-cell leukemia transcription factor interacting protein (HPIP) was initially identified as a co-repressor for PBX-HOX transcriptional complex through a yeast two-hybrid screening of a hematopoietic cDNA library (13). Subsequent studies have demonstrated that HPIP plays a critical role in erythroid differentiation by serving as a target of GATA1, an erythroid lineage-specific transcription factor (14). Recently, increasing studies indicate that HPIP is implicated in carcinogenesis. HPIP has been reported to be overexpressed in many types of cancers, including colorectal cancer (15), astrocytoma (16), thyroid carcinoma (17), breast cancer (18), non-small cell lung cancer (19) and hepatocellular carcinoma (20), and plays an important role in the development and progression of these tumors. In GC, the expression of HPIP was found to be significantly upregulated in tumors compared with that noted in adjacent normal tissues and was found to be positively correlated with tumor size and metastasis (21). HPIP overexpression was found to increase GC cell proliferation by promoting cell cycle progression and to enhance GC cell migration by modulating epithelial-mesenchymal transition (EMT) (21). Although the role of HPIP in promoting GC cell proliferation and activating G1/S transition has been characterized, how HPIP enhances these processes remains unclear. In colorectal cancer and hepatocellular carcinoma $(15,20)$, HPIP has been shown to promote cell proliferation via enhancing AKT/mTORC1 signaling. Due to the important role of the AKT/mTORC1 pathway in the regulation of $4 \mathrm{E}-\mathrm{BP} 1$ phosphorylation, we hypothesized that HPIP may increase GC cell proliferation by activating capdependent translation.

In the present study, we found that HPIP activated cap-dependent translation in an AKT/mTORC1 signalingdependent manner. Furthermore, we showed that inhibition of the activity of the eIF4F complex abolished the ability of HPIP to enhance GC cell proliferation both in vitro and in a xenograft mouse model in vivo, while enhancing activity of the eIF4F complex by silencing 4E-BP1 expression reversed the inhibitory effect of HPIP knockdown on GC cell proliferation. Our data indicate that HPIP promotes GC cell proliferation by enhancing cap-dependent translation.

\section{Materials and methods}

Antibodies and reagents. Antibodies against AKT, pAKT, 4EBP-1, p4EBP-1 (S65), P70S6K, pP70S6K (T389), eIF4E, eIF4G and cyclin D1 were purchased from Cell Signaling Technology, Inc. (Beverly, MA, USA). The anti-tubulin antibody was purchased from Santa Cruz Biotechnology, Inc. (Santa Cruz, CA, USA). Antibody against HPIP was obtained from Proteintech Group, Inc. (Chicago, IL, USA). MK2206 and PP242 were obtained from Selleckchem (Houston, TX, USA). 4EGI-1 was provided by Calbiochem (Darmstadt, Germany).

Cells and cell culture. SGC-7901 and BGC-823 cells were maintained in Dulbecco's modified Eagle's medium (DMEM; Invitrogen Life Technologies, Carlsbad,CA,USA) supplemented with $10 \%$ fetal bovine serum (FBS; Hyclone, Logan, UT, USA) and pen/strep (100 U/ml penicillin and $100 \mathrm{mg} / \mathrm{l}$ streptomycin; Cellgro Mediatech, Inc., Manassas, VA, USA). Cells were cultured in a $37^{\circ} \mathrm{C}$ incubator at $5 \% \mathrm{CO}_{2}$.

To generate stable cell lines with overexpression of HPIP, SGC-7901 and BGC-823 cells were transfected with the pCMV-2B-HPIP vector or pCMV-2B backbone with Lipofectamine 3000 (Promega, Madison, WI, USA) according to the manufacturer's instructions, followed by selection with $600 \mu \mathrm{g} / \mathrm{ml} \mathrm{G} 418$ (Calbiochem) for 2 weeks.

For establishing stable HPIP-knockdown cell lines, SGC-7901 and BGC-823 cells were infected with appropriate amounts of lentiviral particles harboring HPIP shRNA or control shRNA (GeneChem, Co., Ltd., Shanghai, China). Infected cells were selected with $1 \mu \mathrm{g} / \mathrm{ml}$ puromycin (Sigma-Aldrich, St. Louis, MO, USA) for 1 week.

Western blotting. Cells were lysed in RIPA buffer $(50 \mathrm{mM}$ Tris- $\mathrm{HCl}, \mathrm{pH} 7.5,150 \mathrm{mM} \mathrm{NaCl}, 0.1 \%$ SDS, $1 \%$ NP-40, $1 \%$ sodium deoxycholate, $1 \mathrm{mM}$ EDTA, $1 \mathrm{mM}$ EGTA, $1 \mathrm{X}$ protease inhibitor cocktail) on ice. Equal amounts of total protein were resolved by SDS-PAGE and transferred to nitrocellulose membranes (Amersham Biosciences, Piscataway, NJ, USA). After being blocked with 5\% nonfat milk for $1 \mathrm{~h}$ at room temperature, the membranes were probed with primary antibodies overnight at $4^{\circ} \mathrm{C}$, followed by incubation with horseradish peroxidase-conjugated secondary antibodies for $2 \mathrm{~h}$ at room temperature. Detection was performed using ECL $^{\mathrm{TM}}$ Advance western blotting detection kit (GE Healthcare, Buckinghamshire, UK).

siRNA and transient transfections. siRNAs against AKT, Raptor and 4E-BP1 were obtained from Invitrogen Life Technologies. SGC-7901 and BGC-823 cells were transfected with control siRNAs or siRNAs against AKT, Raptor or 4EBP1 using Lipofectamine RNAiMAX (Invitrogen Life Technologies) according to the manufacturer's instructions. For siRNAs against AKT or Raptor, the cells were lysed and subjected to western blotting at $48 \mathrm{~h}$ post-transfection. For siRNAs against 4E-BP1, the cells were lysed and subjected to assays for cap-dependent translation, cell cycle and western blotting at $48 \mathrm{~h}$ post-transfection. For Cell Counting Kit-8 (CCK-8) assay, the cells were seeded into 96-well plates $24 \mathrm{~h}$ after transfection.

Bicistronic luciferase assays. SGC-7901 and BGC-823 cells were transiently transfected with a bicistronic luciferase reporter plasmid, pcDNA3-rLuc-PolioIRES-fLuc, which directs cap-dependent translation of the Renilla luciferase (RL) gene and cap-independent Polio IRES-mediated translation of the firefly (FL) gene. Cells were harvested and analyzed for luciferase activity using Dual-Luciferase reporter assay kit (Promega) at $48 \mathrm{~h}$ post-transfection. Cap-dependent translational activity was determined by calculating the ratio of Renilla/firefly luciferase activity. Assays were performed in triplicate and results are presented as means \pm standard deviation (SD).

$m^{7}$ GTP pull-down assay. Cells were harvested in $\mathrm{m}^{7} \mathrm{GTP}$ lysis buffer $(20 \mathrm{mM}$ Tris, $100 \mathrm{mM} \mathrm{KCl,} 1 \mathrm{mM}$ dithiothreitol, 
$20 \mathrm{mM} \beta$-glycerophosphate, $1 \mathrm{mM}$ phenylmethylsulfonyl fluoride, $1 \mathrm{mM}$ EDTA, $1 \mathrm{mM}$ EGTA, $0.25 \mathrm{mM} \mathrm{Na}_{3} \mathrm{VO}_{4}$, $10 \mathrm{mM} \mathrm{NaF}$ and $1 \mathrm{X}$ protease inhibitor cocktail) on ice. Thirty microliters of $\mathrm{m}^{7} \mathrm{GTP}$-Sepharose beads (GE Healthcare, Chalfont St. Giles, UK) was added to $500 \mu 1$ cell lysate and gently rocked at $4^{\circ} \mathrm{C}$ for $3 \mathrm{~h}$. Precipitates were collected, washed 3 times with $500 \mu$ lysis buffer at $4^{\circ} \mathrm{C}$, and then eluted by boiling in SDS sample buffer for western blotting. The original lysate was performed as a loading control.

Cell cycle analysis. Cells were fixed with $70 \%$ ethanol overnight at $-20^{\circ} \mathrm{C}$, washed twice with ice-cold PBS and then treated with RNase A $(0.1 \mathrm{mg} / \mathrm{ml})$ in PBS for 30 min at $37^{\circ} \mathrm{C}$. Propidium iodide $(50 \mu \mathrm{g} / \mathrm{ml})$ was added to the cellular suspension. DNA contents were determined by a FACSCanto flow cytometer (BD Biosciences, Mississauga, ON, Canada).

Cell proliferation assays. Cells were seeded in 96-well plates at 1,500 cells/well. Cell proliferation was assessed with CCK-8 (Dojindo Laboratories, Tokyo, Japan) assay according to the manufacturer's instructions and the absorbance was determined at a wavelength of $450 \mathrm{~nm}$ using a microplate reader. The optical density (OD) value of the treatment group was normalized to the values from the untreated control group.

Soft agar assays. For anchorage-independent growth assay, the cells were seeded in 6-well plates at 5,000 cells/well, with a bottom layer of $0.35 \%$ low melting point agarose (BD Biosciences) in DMEM/10\% FBS and a top layer of $0.5 \%$ agarose in the same medium. Three weeks later, the number of colonies with diameters $>100 \mu \mathrm{m}$ were counted.

GC xenografts and treatments. Five six-week-old female $\mathrm{BALB} / \mathrm{c}$ nude mice were obtained from the Animal Center of Sun Yat-Sen University (Guangzhou, China). BGC-823 cells $\left(5 \times 10^{6}\right)$ stably overexpressing HPIP or control cells in serum-free medium were injected into the flanks of the nude mice, respectively $(n=12)$. After 10 days, the mice injected with BGC-823 cells stably overexpressing HPIP or control cells were randomly allocated to 2 groups ( $\mathrm{n}=6 /$ group) according to body weight and tumor volume and treated with 4EGI-1 $(50 \mathrm{mg} / \mathrm{kg} / \mathrm{day}$, intraperitoneal injection) or vehicle control. 4EGI-1 was dissolved at $90 \mathrm{mg} / \mathrm{ml}$ in DMSO and stored at $-80^{\circ} \mathrm{C}$. Immediately prior to injection, 4EGI-1 was thawed and diluted 1:9 in saline ( $0.9 \%$ sodium chloride). The tumor volume and the body weight of the mice were monitored at the indicated times. One month after treatment, all mice were sacrificed. Xenograft primary tumors were harvested for western blotting.

Statistical analysis. All data were analyzed using the unpaired Student's t-test with the SPSS statistical software program (version 13.0; SPSS, Inc., Chicago, IL, USA). The data are presented as means \pm SD. P-values $<0.05$ were considered statistically significant.

\section{Results}

HPIP activates cap-dependent translation. Given that HPIP was reported to promote GC cell proliferation and cap-dependent translation promotes cell cycle progression via enhancing the expression of cyclin D1 (21), we hypothesized that HPIP may activate cap-dependent translation in GC cells. To test this hypothesis, the cap-dependent translation rate in the control and the HPIP-overexpressing cells was examined using a dual-luciferase reporter system which can monitor the ratio between cap-dependent (Renilla luciferase) and cap-independent IRES-mediated (firefly luciferase) translation initiation. As shown in Fig. 1A and B, HPIP overexpression promoted cap-dependent translation in both the SGC-7901 and BGC-823 cells. To confirm this result, we further determined the cap-dependent translation rate in the control and HPIP-silenced SGC-7901 and BGC-823 cells. We found that HPIP depletion profoundly suppressed cap-dependent translation (Fig. 1C and D). It is known that cap-dependent translation is regulated by the assembly of the eIF4F complex, which can be monitored with 7-methyl GTP Sepharose bead assay. We subsequently detected the effect of HPIP overexpression on the assembly of the eIF4F complex and found that HPIP overexpression markedly increased the interaction between eIF4E and eIF4G, while suppressed the binding of eIF4E and 4E-BP1 (Fig. 1E), indicating the increased assembly of the eIF4F complex in the HPIP-overexpressing GC cells. Consistent with this result, HPIP knockdown decreased the assembly of the eIF4F complex in both SGC-7901 and BGC-823 cell lines (Fig. 1F). Taken together, these findings suggest that HPIP activates cap-dependent translation by promoting the assembly of the eIF4F complex in GC cells.

HPIP increases cap-dependent translation in an AKT/mTORC1-dependent manner. Cap-dependent translation is mainly regulated by AKT/mTORC1 signaling, which phosphorylates 4E-BP1 and relieves its binding to eIF4E, allowing the formation of the eIF4F complex to initiate translation. As previous studies have demonstrated that HPIP enhances AKT/mTORC1 signaling in thyroid cancer cells and breast cancer cells, we first detected whether HPIP regulates AKT/mTORC1 signaling in GC cells. As shown in Fig. 2A, HPIP overexpression enhanced the phosphorylation of AKT in both the SGC-7901 and BGC-823 cells. Furthermore, the phosphorylation levels of p70S6K and 4E-BP1, downstream substrates of mTORC1, were also significantly increased in the HPIP-overexpressing SGC-7901 and BGC-823 cells (Fig. 2A), indicating that HPIP enhances AKT/mTORC1 signaling in GC cells. We next examined the effect of HPIP knockdown on AKT/mTORC1 signaling and found that HPIP silencing decreased the phosphorylation of AKT, p70S6K and 4E-BP1 (Fig. 2B). To investigate whether AKT/mTORC1 signaling activation is essential for HPIP-mediated cap-dependent translation, control and HPIP-overexpressing GC cells were treated with MK2206 and PP242, which are AKT and $\mathrm{mTORC} 1$ pathway inhibitors, respectively. The results showed that MK2206 and PP242 antagonized the HPIPmediated cap-dependent translation in both the SGC-7901 and BGC-823 cells (Fig. 2C and D). To further confirm the role of $\mathrm{AKT} / \mathrm{mTORC} 1$ signaling activation in this process, we analyzed HPIP-mediated cap-dependent translation after silencing the expression of AKT or Raptor, a specific and essential component of mTORC1. We found that AKT or Raptor depletion abrogated HPIP-mediated cap-dependent translation 

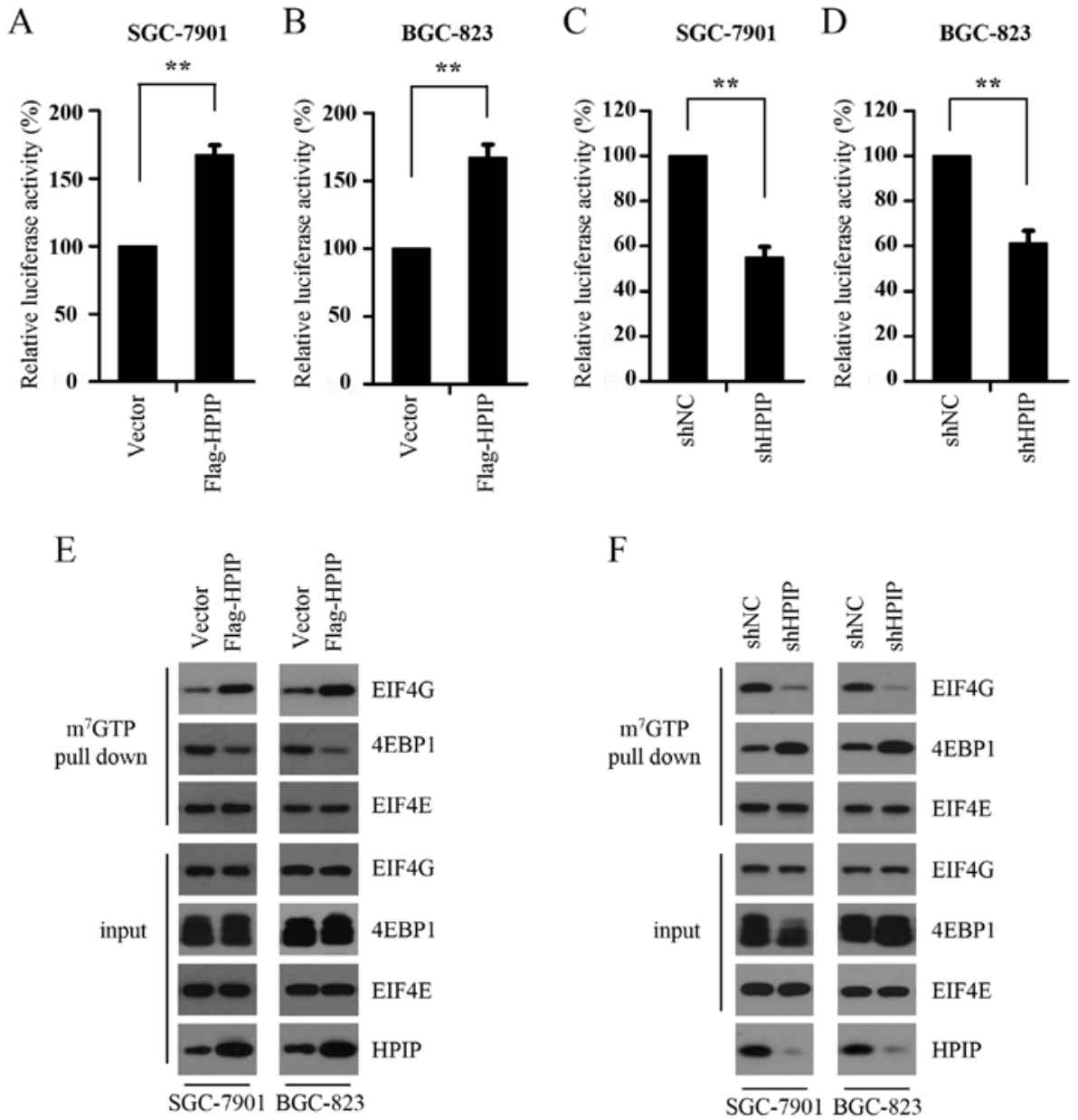

Figure 1. HPIP activates cap-dependent translation. (A-D) Bicistronic luciferase assays. (A) SGC-7901 and (B) BGC-823 cells with stable expression of HPIP or (C) HPIP-knockdown SGC-7901 and (D) BGC-823 cells were transfected with a bicistronic luciferase reporter plasmid. Luciferase activities were measured after $48 \mathrm{~h}$. Cap-dependent translational activity was determined by calculating the ratio of Renilla/firefly luciferase luminescence. Results are shown as means \pm SD of triplicate measurements. ${ }^{* *} \mathrm{P}<0.01$. (E and F) $\mathrm{m}^{7} \mathrm{GTP}$ pull-down assay. (E) Cell lysates from SGC-7901 and BGC-823 cells with stable expression of HPIP or (F) HPIP knockdown SGC-7901 and BGC-823 cells were precipitated with $\mathrm{m}^{7}$ GTP-Sepharose beads followed by western blotting with the indicated antibodies. HPIP, hematopoietic pre-B-cell leukemia transcription factor interacting protein; GC, gastric cancer.

in both the SGC-7901 and BGC-823 cells (Fig. 2E and F). Collectively, these data suggest that AKT/mTORC1 signaling plays an indispensable role in the regulation of HPIP-mediated cap-dependent translation.

HPIP promotes GC cell proliferation by activating cap-dependent translation. Previous studies have reported that HPIP promotes GC cell proliferation by enhancing the expression of cyclin D1 and G1/S transition. Since cap-dependent translation promotes cancer cell cycle progression by initiating the translation of cyclin D1, we ascertained whether cap-dependent translation is required for HPIP-mediated GC cell proliferation. Consistent with the previous results, in the present study, cyclin D1 expression, the proportion of cells in the $\mathrm{S}$ phase and cell proliferation were increased in the HPIP-overexpressing BGC-823 cells. However, the treatment of 4EGI-1, a selective eIF4E/eIF4G interaction inhibitor, abolished the ability of HPIP to enhance the expression of cyclin D1, the proportion of cells in the $\mathrm{S}$ phase and cell proliferation in the BGC-823 cells (Fig. 3A, C and D). Contrary to the increase in cyclin D1 at the protein level by HPIP overexpression and the downregulation by 4EGI-1 treatment, HPIP overexpression and 4EGI-1 treatment had little effect on the mRNA levels of cyclin D1, suggesting that the downregulation of cyclin D1 by 4EGI-1 (Fig. 3A) resulted from the suppression of cyclin D1 translational inhibition rather than its gene transcription (Fig. 3B). Furthermore, soft agar assay showed that HPIP promoted anchorage-independent proliferation of the BGC-823 cells, while 4EGI-1 treatment attenuated the effect of HPIP (Fig. 3E). Taken together, our data suggest that HPIP enhanced GC cell proliferation by activating cap-dependent translation.

HPIP regulates cap-dependent translation and cell proliferation by inactivation of $4 E-B P 1$. Hypo-phosphorylated 4E-BP1 suppresses the assembly of the eIF4F complex by binding competitively to EIF4E, resulting in the inhibition of cap-dependent translation. The above studies demonstrated that HPIP activated cap-dependent translation in an AKT/mTORC1 signaling-dependent manner. Since AKT/mTORC1 signaling enhances cap-dependent translation by phosphorylating 4E-BP1, which leads to the disassociation of 4E-BP1 and EIF4E and contributes to the formation of eIF4F complex, we determined the importance of 4E-BP1 in 
A

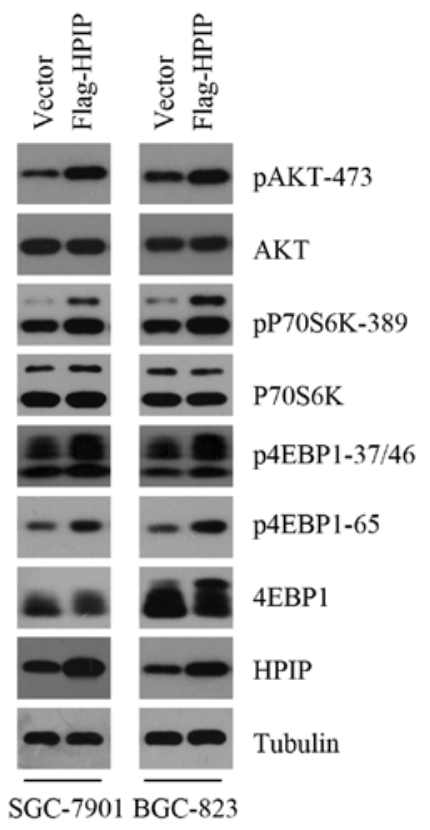

$\mathrm{C}$

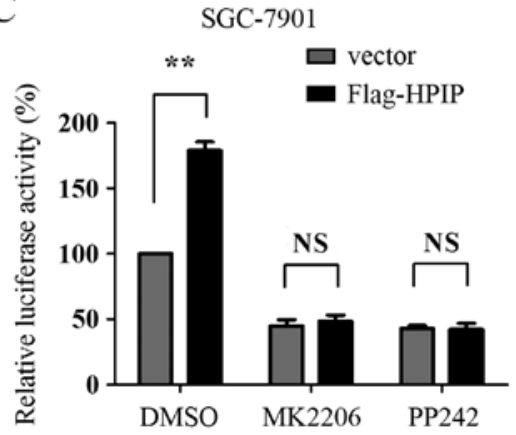

E
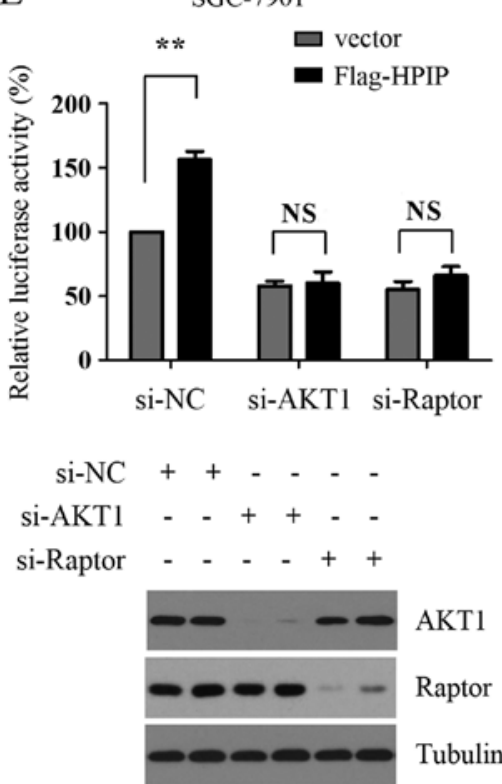

B

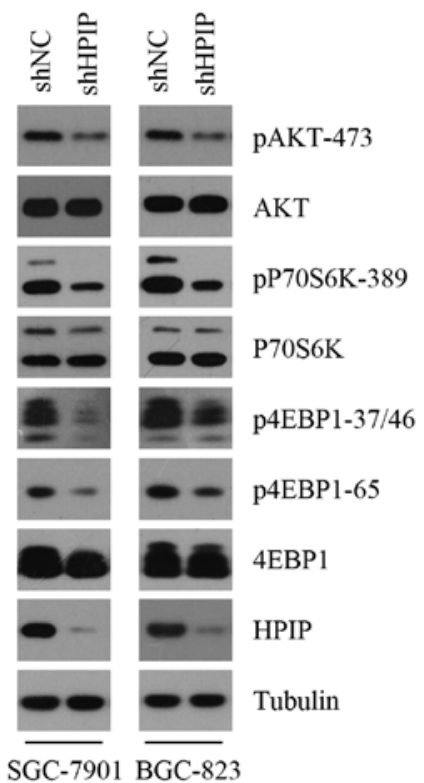

D

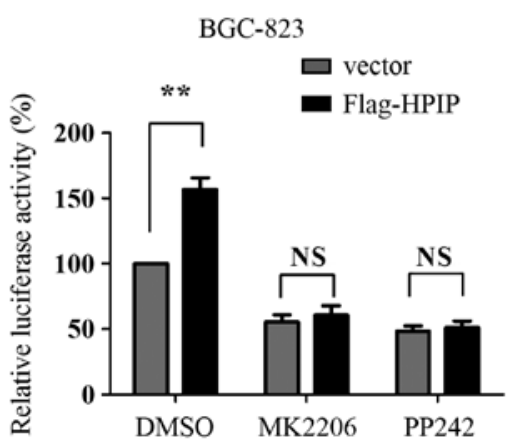

F

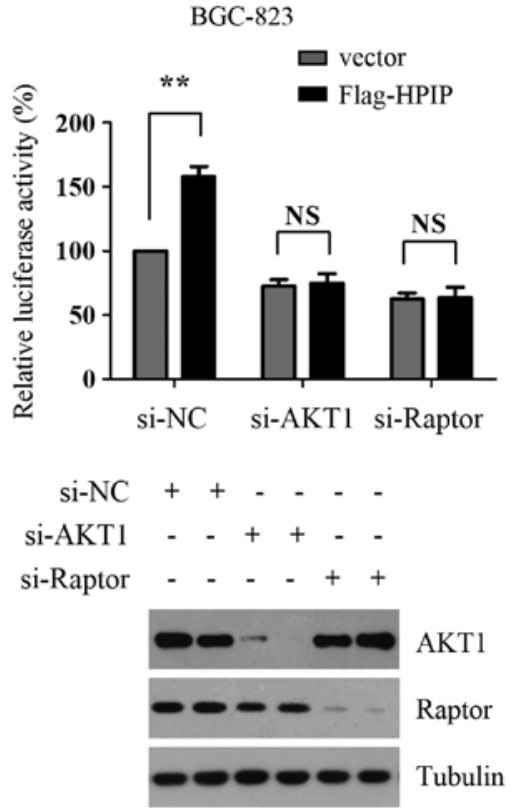

Figure 2. HPIP increases cap-dependent translation in an AKT/mTORC1-dependent manner. (A) Total proteins were extracted from control and GC cell lines (SGC-7901 and BGC-823) with stable expression of HPIP and analyzed by western blotting with the indicated antibodies. Tubulin was used as a loading control. (B) Total proteins were extracted from control and HPIP-knockdown GC cell lines (SGC-7901 and BGC-823) and analyzed by western blotting with the indicated antibodies. Tubulin was used as a loading control. (C) SGC-7901 and (D) BGC-823 cells with stable expression of HPIP were transfected with a bicistronic luciferase reporter plasmid for $24 \mathrm{~h}$, treated with $0.1 \mu \mathrm{M} \mathrm{MK} 2206$ or $0.25 \mu \mathrm{M}$ PP242 for $24 \mathrm{~h}$, and luciferase activity was measured. Results are shown as means \pm SD of triplicate measurements. ${ }^{* * *} \mathrm{P}<0.01$; NS, not significant. (E) SGC-7901 and (F) BGC-823 cells with stable expression of HPIP were transfected with a bicistronic luciferase reporter plasmid and control, AKT or Raptor siRNA for $48 \mathrm{~h}$ and then harvested for luciferase activity analysis. The indicated proteins were detected by western blotting. Results are shown as means \pm SD of triplicate measurements. ${ }^{* *} \mathrm{P}<0.01$; NS, not significant. HPIP, hematopoietic pre-B-cell leukemia transcription factor interacting protein; mTORC1, mammalian target of rapamycin complex 1; GC, gastric cancer. 
A

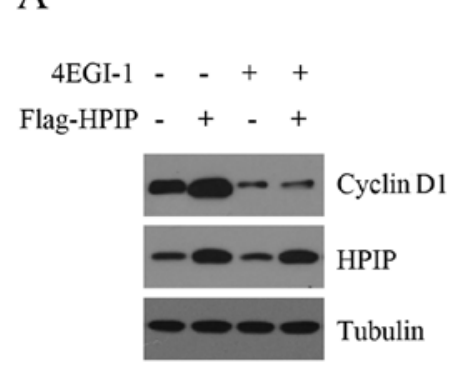

$\mathrm{D}$

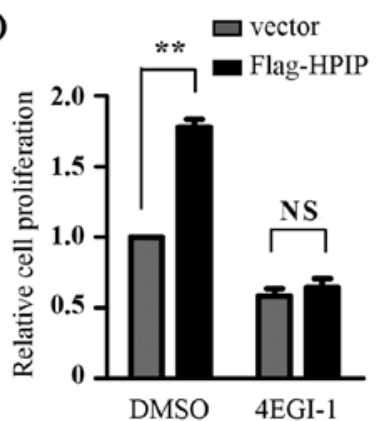

B

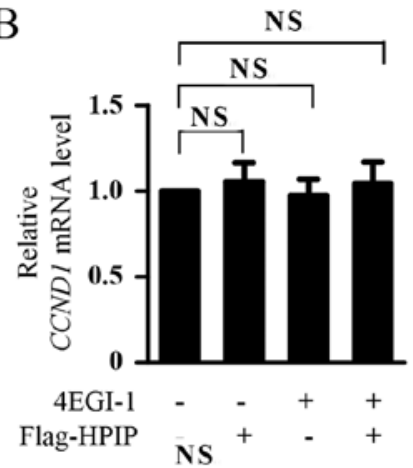

E

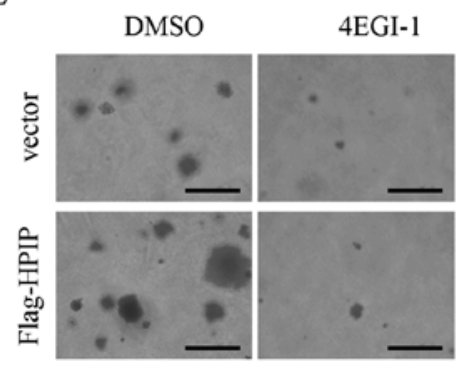

$\mathrm{C}$
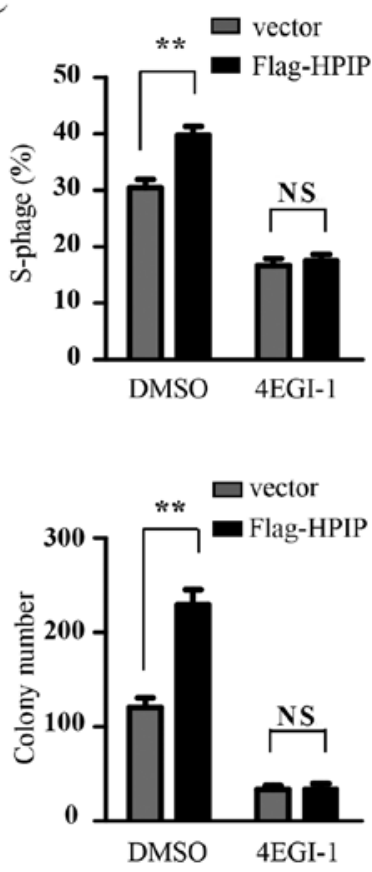

Figure 3. HPIP promotes GC cell proliferation by activating cap-dependent translation. (A) Control and BGC-823 cells with stable expression of HPIP were treated with $50 \mu \mathrm{M} 4 \mathrm{EGI}-1$ for $24 \mathrm{~h}$ and then harvested for western blotting with cyclin D1 antibody. Tubulin was used as a loading control. (B) Control and BGC-823 cells with stable expression of HPIP were treated with DMSO or $50 \mu \mathrm{M}$ 4EGI-1 for $24 \mathrm{~h}$ and then harvested for real-time PCR analysis. All values are normalized to the level of mRNA in the control cells treated with DMSO. Results are shown as means \pm SEM ( $n=3$ ). NS, not significant. (C) Control and BGC-823 cells with stable expression of HPIP were treated with $50 \mu \mathrm{M}$ 4EGI-1 for $24 \mathrm{~h}$ and then harvested for flow cytometry analysis of the cell cycle. Results are shown as means \pm SD of triplicate measurements. ${ }^{* *} \mathrm{P}<0.01$; NS, not significant. (D) Control and BGC-823 cells with stable expression of HPIP were seeded in 96-well plates. On the second day, they were treated with DMSO or $50 \mu \mathrm{M}$ 4EGI-1 for $48 \mathrm{~h}$. Cell number was determined by CCK-8 assay. Results are shown as means $\pm \mathrm{SD}$ of triplicate measurements. ${ }^{* *} \mathrm{P}<0.01$; NS, not significant. (E) Control and BGC-823 cells with stable expression of HPIP were plated in soft agar and colony number was assessed after 3 weeks. Representative colonies in soft agar are shown. Scale bar, $500 \mu \mathrm{M}$. Results are shown as means \pm SD of triplicate measurements. ${ }^{* *} \mathrm{P}<0.01$; NS, not significant. HPIP, hematopoietic pre-B-cell leukemia transcription factor interacting protein; GC, gastric cancer.
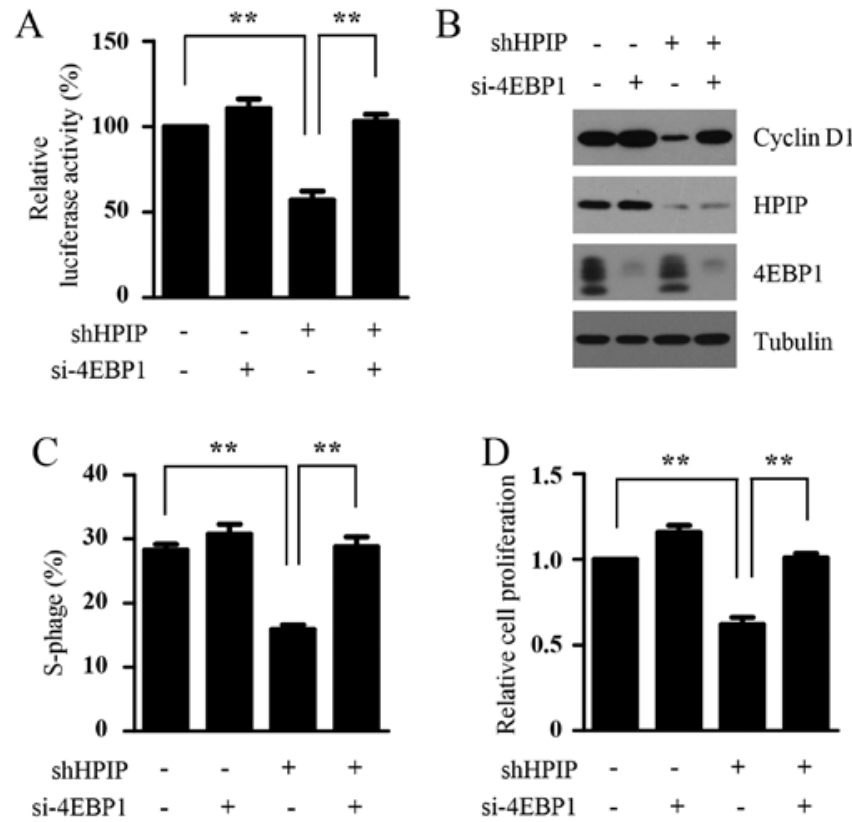

Figure 4. HPIP regulates cap-dependent translation and cell proliferation through inactivation of 4E-BP1. (A) Control and HPIP-knockdown BGC-823 cells were transfected with a bicistronic luciferase reporter plasmid and control or 4E-BP1 siRNA for $48 \mathrm{~h}$ and then harvested for luciferase activity analysis Results are shown as means \pm SD of triplicate measurements. ${ }^{* *} \mathrm{P}<0.01$. (B) Control and HPIP-knockdown BGC-823 cells were transfected with control or 4E-BP1 siRNAs. Forty-eight hours post-transfection, the cells were harvested for western blotting with the cyclin D1 antibody. Tubulin was used as a loading control. (C) Control and HPIP-knockdown BGC-823 cells were transfected with control or 4E-BP1 siRNAs for $48 \mathrm{~h}$ and then harvested for flow cytometry analysis of the cell cycle. Results are shown as means \pm SD of triplicate measurements. ${ }^{* *} \mathrm{P}<0.01$. (D) Control and HPIP-knockdown BGC-823 cells were transfected with control or 4E-BP1 siRNAs. On the second day, they were seeded in 96 -well plates. After $48 \mathrm{~h}$, cell number was determined by CCK-8 assay. Results are shown as means \pm SD of triplicate measurements. ${ }^{* *} \mathrm{P}<0.01$. HPIP, hematopoietic pre-B-cell leukemia transcription factor interacting protein; 4E-BP1, eIF4E-binding protein 1; GC, gastric cancer. 

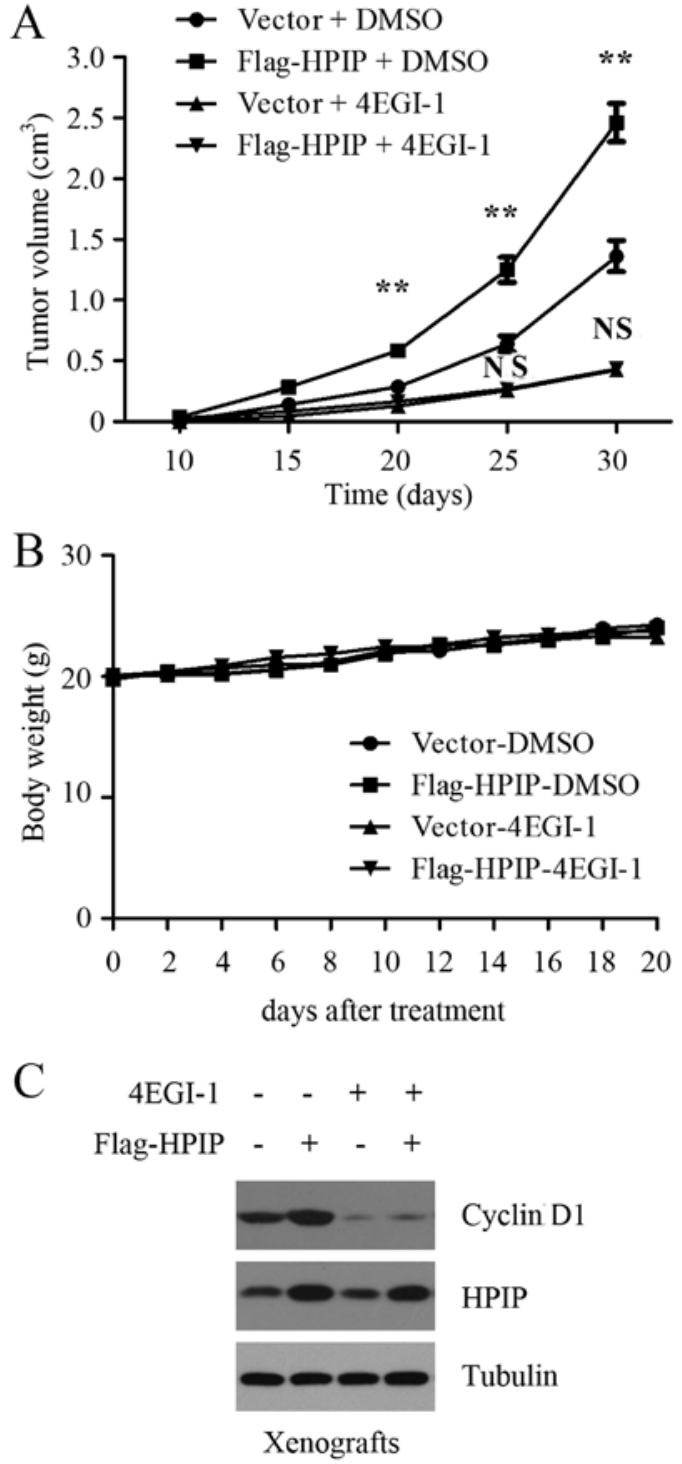

Figure 5. Targeting translation initiation with 4EGI-1 suppresses the ability of HPIP to promote gastric tumor growth in vivo. Control and BGC-823 cells with stable expression of HPIP were injected into nude mice. After 10 days, the mice injected with BGC-823 cells stably overexpressing HPIP or control cells were randomly allocated to 2 groups ( $\mathrm{n}=6$ /group) and treated with vehicle control or 4EGI-1 $(50 \mathrm{mg} / \mathrm{kg} /$ day, intraperitoneal injection). (A) Tumor growth was measured at the indicated times. DMSO groups: ${ }^{* *} \mathrm{P}<0.01$ (vector vs. Flag-HPIP); 4EGI-1 treatment groups: NS, not significant (vector vs. FlagHPIP). (B) Body weights of mice were monitored over a 20-day period. (C) Tumor samples were harvested for western blotting with cyclin D1 antibody. Tubulin was used as a loading control. HPIP, hematopoietic pre-B-cell leukemia transcription factor interacting protein; GC, gastric cancer.

mediating the effects of HPIP on cap-dependent translation and cell proliferation. To test this idea, we silenced the expression of 4E-BP1 in the control and HPIP-depleted BGC-823 cells and detected whether 4E-BP1 depletion attenuated the inhibitory effect of HPIP knockdown on cap-dependent translation, cyclin D1 expression, the proportion of S phase cells and cell proliferation in the GC cells. As shown in Fig. 4A-D, HPIP knockdown suppressed cap-dependent translation, cyclin D1 expression, the proportion of $\mathrm{S}$ phase cells and cell proliferation in the BGC-823 cells, while 4E-BP1 depletion markedly rescued the inhibitory effect. Taken together, our results further confirmed that HPIP promotes cell proliferation by activating cap-dependent translation and suggest that inactivation of 4E-BP1 is essential for HPIP-mediated cap-dependent translation and cell proliferation.

Targeting translation initiation with 4EGI-1 suppresses the ability of HPIP to promote gastric tumor growth in vivo. Having established that HPIP promotes GC cell proliferation by activating cap-dependent translation in vitro, we next examined whether blockage of translation initiation attenuated the ability of HPIP to promote tumor growth in vivo. After the generation of GC xenografts using control and HPIPoverexpressing BGC-823 cells, xenograft mice were randomly grouped and treated with vehicle control or 4EGI-1 for weeks. As expected, HPIP overexpression markedly promoted gastric tumor growth in nude mice, as shown by a representative tumor growth curve (Fig. 5A). Consistent with the results from cell cultures, 4EGI-1 treatment suppressed the ability of HPIP to promote gastric tumor growth in vivo (Fig. 5A). There was no obvious difference in mouse body weights among the groups during the 20 day treatment (Fig. 5B), indicating that the treatment was well tolerated. In addition, the BGC-823 tumors with HPIP overexpression showed increased expression of cyclin D1, but this increase was profoundly reversed by 4EGI-1 treatment (Fig. 5C). These results clearly indicate that HPIP promotes GC cell proliferation by activating cap-dependent translation in vivo.

\section{Discussion}

Aberrantly expressed HPIP plays a critical role in tumorigenesis. Overexpression of HPIP has been found in patients with various types of cancers and is associated with a poor clinical outcome. HPIP promotes cancer cell proliferation by regulating cell cycle progression and cell migration via enhancing EMT. HPIP was reported to increase cell proliferation and migration by regulation of $\mathrm{AKT} / \mathrm{mTORC} 1$ signaling in colorectal cancer and hepatocellular carcinoma $(15,20)$. In breast cancer, HPIP was found to enhance estrogen receptor (ER) signaling and promote tumor growth by activating AKT signaling (18). A recent study demonstrated that the expression of HPIP is higher in GC tissues than that noted in adjacent normal gastric tissues and is significantly correlated with several important clinicopathologic factors. HPIP knockdown profoundly suppressed tumor growth in vitro and in an allograft murine model (21). However, the molecular mechanism by which HPIP promotes GC cell proliferation remains unknown. Given that HPIP activates the AKT/mTORC1 pathway in other cancers and cap-dependent translation is controlled by this pathway, in the present study, we first detected the effect of HPIP on capdependent translation. Using bicistronic luciferase reporter assays, we found that HPIP enhanced cap-dependent translation in GC cells. The results of 7-methyl GTP Sepharose bead assays confirmed this conclusion.

Cap-dependent translation is negatively regulated by 4E-BP1, which inhibits the formation of the eIF4F complex by competing with eIF4G for binding to eIF4E. The phosphorylation of 4E-BP1 controls its activity and is regulated by AKT/mTORC1 signaling, one of the most frequently activated pathways in GC patients (9). mTORC1 promotes cap-dependent translation initiation through relieving 4E-BP1-mediated 
inhibition of eIF4E. Since we found that HPIP enhanced capdependent translation in GC cells, we next determined whether this effect depends on AKT/mTORC1 signaling. Our results indicated that HPIP also increased AKT/mTORC1 signaling in GC cells and suppressing AKT activity with MK2006 or mTORC1 activity with PP242 antagonized HPIP-mediated cap-dependent translation. Inhibition of AKT/mTORC1 signaling by silencing the expression of AKT or Raptor further confirmed this result. These data suggest that activation of AKT/mTORC1 signaling is essential for HPIP-mediated cap-dependent translation.

Cap-dependent translation contributes to cancer cell proliferation by initiating the translation of oncogenes associated with the regulation of cell cycle progression, such as cyclin D1 and c-Myc. cyclin D1 is commonly overexpressed in GC tissues and promotes cell proliferation by driving G1/S phase transition (21). Since previous studies demonstrated that HPIP promotes GC cell proliferation and we found that HPIP enhanced cap-dependent translation in GC cells, we next determined whether HPIP promotes GC cell proliferation by activating cap-dependent translation. We found that the blocking of cap-dependent translation with 4EGI-1, a selective eIF4E/eIF4G interaction inhibitor, abolished the ability of HPIP to promote the expression of cyclin D1 and cell proliferation of GC cells. HPIP depletion suppressed the phosphorylation of $4 \mathrm{E}-\mathrm{BP} 1$, leading to the disruption of the eIF4F complex and inhibition of cap-dependent translation. We released the sequestered eIF4E and rescued the formation of eIF4F by silencing the expression of 4E-BP1 and found that 4E-BP1 knockdown effectively reversed the inhibitory effect of HPIP depletion on cap-dependent translation and cell proliferation in GC, suggesting the importance of 4E-BP1 phosphorylation and cap-dependent translation in mediating the oncogenic role of HPIP. Overexpression of p-4E-BP1 has been shown in $68.4 \%$ of GC tissue samples tested, profoundly higher than in $29 \%$ of normal tissue samples (12). Whether the expression level of HPIP is positively associated with that of phosphorylated 4E-BP1 in clinical tissues remains to be addressed and is a research direction we are currently pursuing. Recently, mTORC1/4E-BP1 signaling has been shown to play an important role in cancer cell migration by translational regulation of Snail, a fundamental regulator in EMT and cancer metastasis (22). Therefore, it will also be interesting to examine if HPIP promotes GC cell migration by enhancing the translational initiation of Snail in the future.

Our results also suggest that direct targeting of capdependent translation with 4EGI-1 abrogates the ability of HPIP to promote gastric tumor growth in vivo, indicating that targeting the eIF4F complex may provide a promising treatment strategy for GC patients with elevated HPIP expression. Indeed, several translation initiation inhibitors, including eIF4E-specific antisense oligonucleotides (ASOs) and silvestrol that suppresses cap-dependent translation by inhibiting the activity of eIF4A, have recently exerted effective antitumor effects with limited toxicity in mice (23-26). In addition, targeting mTORC1 presents an alternative strategy for blocking cap-dependent translation. However, everolimus (RAD001), an oral inhibitor of mTORC1, failed to show improved survival in the phase III GRANITE-1 study for advanced GC patients (2). Weak or only transient inhibition of mTORC1-mediated 4E-BP1 phosphorylation and capdependent translation by everolimus may explain the limited anticancer efficacy in clinical trials $(27,28)$. In the present study, we also found that $10 \mathrm{nM}$ RAD001 treatment had little effect on cap-dependent translation in GC cells and failed to inhibit HPIP-mediated cap-dependent translation (data not shown). ATP-competitive mTOR kinase inhibitors, including OSI-027, PP242, AZD8055 and Torin 1, all strongly inhibit mTORC1-mediated 4E-BP1 phosphorylation and exhibit significant antitumor activity in many cancer xenograft models (29-32). Several of these have entered clinical trials and hold potential as a future strategy for cancer therapeutics (33-35).

\section{Acknowledgements}

This study was supported by the Research Program of Science and Technology at Universities of Inner Mongolia Autonomous Region (NJZY11127 to Y.Z.).

\section{References}

1. Siegel R, Naishadham D and Jemal A: Cancer statistics, 2013. CA Cancer J Clin 63: 11-30, 2013.

2. Ohtsu A, Ajani JA, Bai YX, Bang YJ, Chung HC, Pan HM, Sahmoud T, Shen L, Yeh KH, Chin K, et al: Everolimus for previously treated advanced gastric cancer: results of the randomized, double-blind, phase III GRANITE-1 study. J Clin Oncol 31: 3935-3943, 2013.

3. Kong J and Lasko P: Translational control in cellular and developmental processes. Nat Rev Genet 13: 383-394, 2012.

4. Spilka R, Ernst C, Mehta AK and Haybaeck J: Eukaryotic translation initiation factors in cancer development and progression. Cancer Lett 340: 9-21, 2013.

5. Pettersson F, Del Rincon SV and Miller WH Jr: Eukaryotic translation initiation factor $4 \mathrm{E}$ as a novel therapeutic target in hematological malignancies and beyond. Expert Opin Ther Targets 18: 1035-1048, 2014.

6. Pelletier J, Graff J, Ruggero D and Sonenberg N: Targeting the eIF4F translation initiation complex: a critical nexus for cancer development. Cancer Res 75: 250-263, 2015.

7. Chiarini F, Evangelisti C, McCubrey JA and Martelli AM: Current treatment strategies for inhibiting mTOR in cancer. Trends Pharmacol Sci 36: 124-135, 2015.

8. Liang S, Guo R, Zhang Z, Liu D, Xu H, Xu Z, Wang X and Yang L: Upregulation of the eIF4E signaling pathway contributes to the progression of gastric cancer, and targeting eIF4E by perifosine inhibits cell growth. Oncol Rep 29: 2422-2430, 2013.

9. Yang W, Raufi A and Klempner SJ: Targeted therapy for gastric cancer: molecular pathways and ongoing investigations. Biochim Biophys Acta 1846: 232-237, 2014.

10. Yang HY, Xue LY, Xing LX, Wang J, Wang JL, Yan X and Zhang XH: Putative role of the mTOR/4E-BP1 signaling pathway in the carcinogenesis and progression of gastric cardiac adenocarcinoma. Mol Med Rep 7: 537-542, 2013.

11. Tapia O, Riquelme I, Leal P, Sandoval A, Aedo S, Weber H, Letelier P, Bellolio E, Villaseca M, Garcia P, et al: The $\mathrm{PI} 3 \mathrm{~K} / \mathrm{AKT} / \mathrm{mTOR}$ pathway is activated in gastric cancer with potential prognostic and predictive significance. Virchows Arch 465: 25-33, 2014.

12. Sun DF, Zhang YJ, Tian XQ, Chen YX and Fang JY: Inhibition of mTOR signalling potentiates the effects of trichostatin $\mathrm{A}$ in human gastric cancer cell lines by promoting histone acetylation. Cell Biol Int 38: 50-63, 2014.

13. Abramovich C, Shen WF, Pineault N, Imren S, Montpetit B, Largman $\mathrm{C}$ and Humphries RK: Functional cloning and characterization of a novel nonhomeodomain protein that inhibits the binding of PBX1-HOX complexes to DNA. J Biol Chem 275: 26172-26177, 2000.

14. Manavathi B, Lo D, Bugide S, Dey O, Imren S, Weiss MJ and Humphries RK: Functional regulation of pre-B-cell leukemia homeobox interacting protein 1 (PBXIP1/HPIP) in erythroid differentiation. J Biol Chem 287: 5600-5614, 2012. 
15. Feng Y, Xu X, Zhang Y, Ding J, Wang Y, Zhang X, Wu Z, Kang L, Liang Y, Zhou L, et al: HPIP is upregulated in colorectal cancer and regulates colorectal cancer cell proliferation, apoptosis and invasion. Sci Rep 5: 9429, 2015.

16. van Vuurden DG, Aronica E, Hulleman E, Wedekind LE, Biesmans D, Malekzadeh A, Bugiani M, Geerts D, Noske DP, Vandertop WP, et al: Pre-B-cell leukemia homeobox interacting protein 1 is overexpressed in astrocytoma and promotes tumor cell growth and migration. Neuro-oncol 16: 946-959, 2014.

17. Wang SC, Chai DS, Chen CB, Wang ZY and Wang L: HPIP promotes thyroid cancer cell growth, migration and EMT through activating PI3K/AKT signaling pathway. Biomed Pharmacother 75: 33-39, 2015.

18. Wang X, Yang Z, Zhang H, Ding L, Li X, Zhu C, Zheng Y and Ye Q: The estrogen receptor-interacting protein HPIP increases estrogen-responsive gene expression through activation of MAPK and AKT. Biochim Biophys Acta 1783: 1220-1228, 2008

19. Pan J, Qin Y and Zhang M: HPIP promotes non-small cell lung cancer cell proliferation, migration and invasion through regulation of the Sonic hedgehog signaling pathway. Biomed Pharmacother 77: 176-181, 2016.

20. Xu X, Fan Z, Kang L, Han J, Jiang C, Zheng X, Zhu Z, Jiao H, Lin J, Jiang K, et al: Hepatitis B virus $X$ protein represses miRNA-148a to enhance tumorigenesis. J Clin Invest 123: 630-645, 2013

21. Feng Y, Li L, Zhang X, Zhang Y, Liang Y, Lv J, Fan Z, Guo J, Hong T, Ji B, et al: Hematopoietic pre-B cell leukemia transcription factor interacting protein is overexpressed in gastric cancer and promotes gastric cancer cell proliferation, migration, and invasion. Cancer Sci 106: 1313-1322, 2015.

22. Cai W, Ye Q and She QB: Loss of 4E-BP1 function induces EMT and promotes cancer cell migration and invasion via cap-dependent translational activation of snail. Oncotarget 5: 6015-6027, 2014

23. Graff JR, Konicek BW, Vincent TM, Lynch RL, Monteith D, Weir SN, Schwier P, Capen A, Goode RL, Dowless MS, et al: Therapeutic suppression of translation initiation factor eIF4E expression reduces tumor growth without toxicity. J Clin Invest 117: 2638-2648, 2007

24. Cencic R, Carrier M, Galicia-Vázquez G, Bordeleau ME, Sukarieh R, Bourdeau A, Brem B, Teodoro JG, Greger H, Tremblay ML, et al: Antitumor activity and mechanism of action of the cyclopenta $[b]$ benzofuran, silvestrol. PLoS One 4: e5223, 2009.

25. Blagden SP and Willis AE: The biological and therapeutic relevance of mRNA translation in cancer. Nat Rev Clin Oncol 8 . 280-291, 2011
26. Wang H, Huang F, Wang J, Wang P, Lv W, Hong L, Li S and Zhou J: The synergistic inhibition of breast cancer proliferation by combined treatment with 4EGI-1 and MK2206. Cell Cycle 14: 232-242, 2015

27. Choo AY, Yoon SO, Kim SG, Roux PP and Blenis J: Rapamycin differentially inhibits S6Ks and 4E-BP1 to mediate cell-type-specific repression of mRNA translation. Proc Natl Acad Sci USA 105: 17414-17419, 2008.

28. Choo AY and Blenis J: Not all substrates are treated equally: implications for mTOR, rapamycin-resistance and cancer therapy. Cell Cycle 8: 567-572, 2009.

29. Bhagwat SV, Gokhale PC, Crew AP, Cooke A, Yao Y, Mantis C, Kahler J, Workman J, Bittner M, Dudkin L, et al: Preclinical characterization of OSI-027, a potent and selective inhibitor of mTORC1 and mTORC2: distinct from rapamycin. Mol Cancer Ther 10: 1394-1406, 2011.

30. Feldman ME, Apsel B, Uotila A, Loewith R, Knight ZA, Ruggero D and Shokat KM: Active-site inhibitors of mTOR target rapamycin-resistant outputs of mTORC1 and mTORC2. PLoS Biol 7: e38, 2009.

31. Chresta CM, Davies BR, Hickson I, Harding T, Cosulich S, Critchlow SE, Vincent JP, Ellston R, Jones D, Sini P, et al: AZD8055 is a potent, selective, and orally bioavailable ATP-competitive mammalian target of rapamycin kinase inhibitor with in vitro and in vivo antitumor activity. Cancer Res 70 : 288-298, 2010

32. Thoreen CC, Kang SA, Chang JW, Liu Q, Zhang J, Gao Y, Reichling LJ, Sim T, Sabatini DM and Gray NS: An ATP-competitive mammalian target of rapamycin inhibitor reveals rapamycin-resistant functions of mTORC1. J Biol Chem 284: 8023-8032, 2009.

33. Benjamin D, Colombi M, Moroni C and Hall MN: Rapamycin passes the torch: a new generation of mTOR inhibitors. Nat Rev Drug Discov 10: 868-880, 2011.

34. Naing A, Aghajanian C, Raymond E, Olmos D, Schwartz G, Oelmann E, Grinsted L, Burke W, Taylor R, Kaye S, et al: Safety, tolerability, pharmacokinetics and pharmacodynamics of AZD8055 in advanced solid tumours and lymphoma. Br J Cancer 107: 1093-1099, 2012.

35. Asahina H, Nokihara H, Yamamoto N, Yamada Y, Tamura Y, Honda K, Seki Y, Tanabe Y, Shimada H, Shi X, et al: Safety and tolerability of AZD8055 in Japanese patients with advanced solid tumors; a dose-finding phase I study. Invest New Drugs 31: 677-684, 2013 